\title{
Reflets
}

Revue d'intervention sociale et communautaire

\section{Organisation sociale de la mise en liberté provisoire : des effets de profilage?}

\section{Françoise Vanhamme}

Volume 22, numéro 1, printemps 2016

Profilage social et judiciarisation de la marginalité

URI : https://id.erudit.org/iderudit/1037162ar

DOI : https://doi.org/10.7202/1037162ar

Aller au sommaire du numéro

Éditeur(s)

Reflets, Revue d'intervention sociale et communautaire

ISSN

1203-4576 (imprimé)

1712-8498 (numérique)

Découvrir la revue

Citer cet article

Vanhamme, F. (2016). Organisation sociale de la mise en liberté provisoire : des effets de profilage? Reflets, 22(1), 28-55. https://doi.org/10.7202/1037162ar
Résumé de l'article

La présente contribution se veut une synthèse des récents travaux que nous avons menés sur la détermination judiciaire d'une mise en liberté ou en détention provisoire. Elle vise à en montrer l'organisation sociale, en l'occurrence comment les raisonnements qui sous-tendent cette décision sont ancrés dans des nécessités socioprofessionnelles. En effet, dans un contexte balisé par le droit et le contrôle politique et médiatique, un principe de précaution partagé s'articule à une approche globale de défense sociale et mène à une escalade de conditions de libération (cautionnement en argent, recours à une personne garante, encadrement institutionnel) auxquelles les justiciables socialement marginalisés ne peuvent que difficilement répondre. La détention provisoire peut en devenir plus fréquente pour ce groupe. Le profilage social qui s'en esquisse serait ainsi un effet des pratiques en contexte.
Tous droits réservés (C) Reflets, Revue d’intervention sociale et communautaire, 2016
Ce document est protégé par la loi sur le droit d'auteur. L’utilisation des services d’Érudit (y compris la reproduction) est assujettie à sa politique d'utilisation que vous pouvez consulter en ligne.

https://apropos.erudit.org/fr/usagers/politique-dutilisation/ 


\section{Organisation sociale de la mise en liberté provisoire : des effets de profilage?}

Françoise Vanhamme

Professeure agrégée

Département de criminologie, Université d'Ottawa

\section{Résumé}

La présente contribution se veut une synthèse des récents travaux que nous avons menés sur la détermination judiciaire d'une mise en liberté ou en détention provisoire. Elle vise à en montrer l'organisation sociale, en l'occurrence comment les raisonnements qui sous-tendent cette décision sont ancrés dans des nécessités socioprofessionnelles. En effet, dans un contexte balisé par le droit et le contrôle politique et médiatique, un principe de précaution partagé s'articule à une approche globale de défense sociale et mène à une escalade de conditions de libération (cautionnement en argent, recours à une personne garante, encadrement institutionnel) auxquelles les justiciables socialement marginalisés ne peuvent que difficilement répondre. La détention provisoire peut en devenir plus fréquente pour ce groupe. Le profilage social qui s'en esquisse serait ainsi un effet des pratiques en contexte.

Mots clés : justice criminelle, détention provisoire, détermination de la peine comme pratique sociale, disparités, justice actuarielle, sociologie cognitive.

\section{Abstract}

This paper offers a synthesis of several studies we recently conducted on the judicial determination of interim release or 
detention. It aims to show the social organization of these judicial determinations, notably how the rationales underlying them are embedded in social and professional requirements. Indeed, in a context controlled by law, policy and media, a precaution principle working together with a social defense approach leads to an escalation of the conditions for bail. Socially disadvantaged people hardly could manage these conditions, meaning that they could more likely receive pretrial detention. Such social profiling, as emerging, can be understood as an effect of practices in context.

Key words: criminal justice, remand, sentencing as a social practice, disparities, actuarial justice, cognitive sociology.

\section{Introduction}

"...une détention

provisoire tend

à déstabiliser les

personnes qui

l'expérimentent; en

conséquence, celles qui sont déjà vulnérables seront particulièrement touchées. "
C'est presque un truisme que de mentionner que les personnes marginales ou vulnérables sur le plan économique, social ou de la santé mentale sont surreprésentées dans les prisons. Ce constat récurrent constitue d'ailleurs la base de nombreux travaux et débats pénologiques. Souvent, les études qui s'intéressent à ces tendances traitent de la population qui est détenue pour l'exécution d'une peine. Or, les établissements carcéraux hébergent aussi un autre groupe de personnes détenues en vertu d'un mandat judiciaire, quoiqu'aucun jugement définitif sur la culpabilité ou sur la peine n'ait encore été prononcé à leur encontre : ce sont les prévenus. Leur détention est dénommée "provisoire » ou "avant jugement " et, au sein de cette mesure, les groupes sociaux marginaux ou vulnérables sont davantage concernés.

En effet, une détention provisoire tend à déstabiliser les personnes qui l'expérimentent; en conséquence, celles qui sont déjà vulnérables seront particulièrement touchées. En ce qui concerne les composantes de cette fragilisation au Canada, différents auteurs ont d'abord souligné les conditions de l'encadrement carcéral d'un prévenu. Celui-ci connaît généralement une surveillance plus stricte qu'un condamné, un suivi médical plus restreint et un plus grand désœuvrement en raison de la (probable) courte 
"...plus de la moitié des personnes incarcérées dans les établissements pénitentiaires des provinces et territoires sont des prévenus..." durée du séjour en prison, qui ne prête guère à la mise en œuvre de programmes d'activité ou d'intervention (McLean, 1995; Kellough et Wortley 2002; John Howard, 2009). La détention avant jugement a également un coût psychologique important pour un prévenu. Notamment, la rupture de la vie sociale et la communication restreinte avec l'extérieur, l'immersion dans l'environnement carcéral et sa promiscuité, ainsi que l'incertitude de l'avenir créent angoisse et stress (McLean, 1995; CNBES, 2000; Webster, 2009). Il s'y ajoute un coût social substantiel en raison de sérieuses perturbations dans les liens sociaux, associées à une perte probable de réputation et d'emploi (Trotter, 1999; John Howard, 2009). Par ailleurs, une mise en détention provisoire peut produire une série d'effets sur les suites de la procédure judiciaire. Du fait même de son incarcération, un prévenu préparera moins facilement sa défense; il plaidera aussi plus souvent coupable qu'un accusé laissé en liberté (Friedland, 1965; Koza et Doob, 1974; Trotter, 1999). Quant à sa sentence, elle consistera plus fréquemment en un emprisonnement et celui-ci sera souvent d'une durée plus longue que celle d'un accusé laissé en liberté (Cousineau, 1995; Kellough, 1996; Webster, 2009). La recherche au Canada met clairement en relief que ces différents coûts s'amplifient pour les groupes sociaux marginaux ou vulnérables sur le plan culturel, physique, mental ou économique (Garceau, 1990; Landreville et Laberge, 1992; Kellough et Wortley, 2002; Crocker, 2007; John Howard, 2009).

Ces différents constats deviennent plus aigus encore lorsqu'il apparaît qu'au Canada, le nombre de détentions provisoires et leur proportion dans la population carcérale ont fortement crû ces dernières décennies. Actuellement, plus de la moitié des personnes incarcérées dans les établissements pénitentiaires des provinces et territoires sont des prévenus, comparativement à environ un quart deux décennies plus tôt (Porter et Calverley, 2011) ${ }^{1}$. À cet égard, Webster, Doob et Myers ont montré que cet emballement n'a pas de lien avec une augmentation de la criminalité enregistrée, puisque les chiffres de la criminalité sont à la baisse depuis les années 1990; il n'est pas non plus le fait d'une délinquance plus violente qui astreindrait à davantage de mises 
en détention. Dès lors, étant donné que cette hausse ne peut être attribuée à des variations dans la criminalité, les mécanismes y menant sont à chercher à l'intérieur même du système pénal, dans ses propres opérations (Webster, Doob et Myers, 2009). Il apparait ainsi utile d'interroger les processus judiciaires qui mènent à une décision de mise en liberté ou en détention provisoire. Le présent article montrera ceux qui, parmi ces derniers, contribuent à un traitement différencié dans lequel les personnes vulnérables risquent davantage une mise en détention.

À la suite d'un bref rappel du cadre conceptuel et des dispositifs de recherche dont est issue la présente étude (I), nous donnerons un aperçu des dispositions juridiques relatives à la décision de mise en liberté ou en détention provisoire (II), pour permettre de mieux comprendre la suite du texte. Nous dégagerons ensuite deux grandes lignes de force transversales qui se dégagent de notre enquête : la prémisse de précaution qui sous-tend les raisonnements (III), et l'emballement des exigences posées pour une remise en liberté (IV), lesquelles sont susceptibles de toucher particulièrement les populations marginales ou vulnérables $(\mathrm{V})$.

\section{Une approche sociocognitive de la décision}

La présente contribution se fonde sur une synthèse de nos récents travaux (Labelle et Vanhamme, 2015; Vanhamme, 2015²) dans lesquels nous avons cherché à comprendre les motifs, raisonnements et nécessités qui sous-tendent l'activité menant à une décision de mise en liberté ou en détention provisoire du point de vue de la poursuite et du tribunal. Selon la perspective sociocognitive que nous y avons adoptée (Cicourel, 1979), un acteur judiciaire acquiert, dans le cours de ses activités, un savoir pratique typique portant sur les nécessités de son environnement professionnel autant sur le plan juridique que culturel, organisationnel ou social. Ce savoir multiple lui permet d'interpréter en contexte la situation dont il est saisi et d'en délimiter les enjeux, pour ensuite agir en conséquence d'une façon qu'il estime adéquate à son groupe d'appartenance (Sudnow, 1965; Hutton, 2006;Vanhamme, 2009). 
"...qu'une décision judiciaire... est le fruit d'une interprétation de la norme juridique articulée au contexte dans lequel ils évoluent."
La décision s'organise de ce fait socialement. Cette perspective permet notamment d'éclairer la relative conformité qui a été observée entre les décisions des différents magistrats d'un tribunal (Beyens, 2000). Il s'en déduit aussi qu'une décision judiciaire ne peut pas être appréhendée comme une simple application du droit à la situation. Elle ne peut pas davantage être uniquement rapportée aux caractéristiques individuelles des magistrats : elle est le fruit d'une interprétation de la norme juridique articulée au contexte dans lequel ils évoluent. Ce sont ces bases typiques de raisonnement en contexte que nous avons interrogées.

Pour ce faire, plusieurs dispositifs d'entretiens ouverts ont été menés en cascade durant l'année 2012. Tous visaient le même objectif de recherche, c'est-à-dire de dégager ces nécessités typiques qui sous-tendent l'activité décisionnelle (de réquisition, de jugement). Comme nous allons le voir, ces différents dispositifs ont visé une diversification à la fois intragroupe (selon le ressort, le statut, l'ancienneté dans la fonction et le genre) et intergroupe (par les différentes fonctions consultées) tendant à atteindre une saturation des données (Pires, 1997). Deux régions judiciaires aux caractéristiques socioéconomiques comparables ont été observées. Bien que géographiquement proches, ces régions appartiennent à deux provinces différentes ${ }^{3}$ qui comptent parmi celles qui alimentent de façon importante le nombre des détentions provisoires au Canada. En 2013-2014, la proportion des prévenus par rapport au nombre total des détenus (avant et après condamnation) s'y situait dans une fourchette de 10 de part et d'autre de la tendance nationale (54\%).

Dans la province A, un premier entretien de groupe d'une matinée a réuni quatre juges (un juge de la Cour supérieure, un juge d'instance provinciale et deux juges de paix), ainsi que trois procureurs de la poursuite, trois avocats de la défense et un policier; à la suite, deux juges et un procureur ont participé à un entretien privé d'approfondissement (Labelle, 2012;Vanhamme, 2015).Dans la province $\mathrm{B}$, sept procureurs de la Couronne se sont également rencontrés lors d'un entretien de groupe d'une matinée (Labelle et Vanhamme, 2015). De plus, cinq entretiens individuels y ont été menés avec des juges (Vanhamme, 2015) ${ }^{4}$. Bien que la taille 
des différents échantillons soit restreinte, ces entretiens menés en profondeur ont fourni des données riches et variées permettant à ce stade d'élaborer pour chacun des dispositifs un modèle d'interprétation de type exploratoire.

À cet effet, les différents corpus de données ont fait l'objet d'une analyse spécifique. Chacune d'entre elles s'inscrit toutefois dans une même stratégie interprétative selon laquelle il convient, pour comprendre un phénomène social, de partir du sens que les personnes apportent à ce qu'ils font (Lessard-Hébert, Goyette et Boutin,1997), compte tenu de leurs interactions avec les autres et de leur contexte conformément à notre approche sociocognitive. Dans cet esprit, nous n'avons pas considéré que tous les acteurs pensent de façon identique, et n'avons donc pas négligé les dissensions entre les discours. Au contraire, nous avons examiné les divergences qui ont pu apparaitre, afin d'en dégager, le cas échéant, des raisonnements et nécessités qui les animent en commun : à quels enjeux répondent ces diverses prises de position? Chaque étude a ainsi identifié un ensemble de logiques qui émanent de façon consistante et récurrente des différents discours recueillis.

Comme il a déjà été mentionné, l'étude ci-dessous est issue d'une démarche de synthèse de ces multiples éclairages sur le processus judiciaire en matière de mise en liberté ou de détention

"...nous avons

cherché, ...les raisonnements, motifs et nécessités qui rallient, confrontent ou opposent les procureurs de la poursuite et les juges sur le sujet." provisoire. Selon une même approche, nous avons cherché, à partir de nos résultats précédents, les raisonnements, motifs et nécessités qui rallient, confrontent ou opposent les procureurs de la poursuite et les juges sur le sujet. La diversification des échantillons et la profondeur des données ont permis d'alimenter une description dense et complexe du phénomène à l'étude (Geertz, 1998) et d'en produire un modèle d'interprétation pouvant prétendre à une saturation théorique satisfaisante, au sens où les dernières données examinées n'y ont plus ajouté de propriété nouvelle (Pires, 1997). Sa validité est de surcroît soutenue par les expériences et les avis mutuels rapportés par les divers participants et participantes relativement aux autres rôles et fonctions que les leurs. La raison pour laquelle les positions de la défense n'y apparaitront qu'en filigrane, au travers des points de vue des procureurs de la Couronne et des juges, est double. En effet, d'une part, le nombre 
d'avocats de la défense interviewés est trop restreint pour que nous puissions en dégager des lignes généralisables et, d'autre part, une décision acceptable se négocie en fin de compte principalement entre les procureurs de la poursuite et les juges. De même, la présente contribution se centre principalement sur l'enquête sur la mise en liberté, là où se construit cette décision du tribunal. De cette synthèse ont ainsi émergé d'importants enjeux et logiques qui sont au cœur de ce processus judiciaire et qui interpellent le droit pour tous, hors juste cause, à une mise en liberté assortie d'une caution raisonnable.

\section{Le contexte juridique : le principe du droit à la liberté}

La Charte canadienne des droits et libertés de 1982 garantit en effet à chacun le droit à la liberté et à la sécurité de sa personne; il ne peut être porté atteinte à ce droit qu'en respect des principes de justice fondamentale (art. 7). Cette garantie constitutionnelle est clairement étayée quand la Charte atteste de la protection contre la détention ou l'emprisonnement arbitraire (art. 9 et 10) et quand elle assure du droit, hors juste cause, à une mise en liberté assortie d'une caution raisonnable (art. 11).

Ce principe du droit à la liberté sous-tend tous les stades de la procédure qui peut mener à une détention avant jugement. Lorsque la police trouve un probable contrevenant, elle ne peut l'arrêter que pour des raisons d'intérêt public (p. ex. gravité du fait, protection de la preuve, risque de commission d'un nouveau délit) (Code criminel, art. 495). À la suite d'une telle arrestation, l'agent ou son supérieur peuvent encore le libérer et délivrer une promesse de comparaitre assortie d'une caution ou de conditions. Si la police décide malgré tout du maintien de la mise sous garde, elle doit présenter le justiciable devant un juge de paix dans les 24 heures de l'arrestation (Code criminel, art. 503). C'est la Couronne qui détient les clés de cette première comparution contradictoire. Soit la Couronne consent à la mise en liberté avec ou sans conditions, en fonction des échanges qu'elle aura déjà tenus avec l'avocat de la 
défense; le juge délivrera dans ce cas une promesse à comparaitre. Soit la Couronne s'oppose à la libération, et le juge en prend acte; c'est le point de départ de la détention provisoire. L'objection de la poursuite implique la tenue d'une audience d'enquête sur la mise en liberté (dite aussi "sur cautionnement»), si l'accusé ne plaide pas coupable et s'il souhaite cette audience; celle-ci doit se tenir dans les trois jours. Durant ce délai, les parties entrent en négociation pour élaborer les modalités d'une libération. Par exemple, la Couronne peut à ce stade demander une évaluation du prévenu; la défense, elle, peut chercher une personne garante ou une caution en argent. Pour arriver à de telles fins, le délai de trois jours peut être prolongé avec l'accord du justiciable (Code criminel, art. 516).Alors, les parties, fortes d'un accord, se présentent au tribunal où elles soumettent le cas au juge.

En concordance avec le droit à la liberté mentionné, la loi stipule explicitement que le juge doit en principe libérer le prévenu (Code criminel, art. 515[1]). Il peut ainsi décider (Code criminel, art. $515[2])$ :

- d'une libération simple - éventualité douteuse si la Couronne s'y est opposée jusque-là;

- d'une libération assortie d'une caution en argent. Celle-ci peut consister en un dépôt d'argent comptant ou en un engagement à payer, dont le montant ne peut être élevé au point d'équivaloir à une mise en détention $(R$. v. Garrington, 1973); et/ou

- d'une libération accompagnée de conditions. Ces dernières peuvent viser le contrôle de la personne par un policier, consister en une interdiction de communiquer avec les témoins ou les victimes, ou toute autre interdiction ou obligation raisonnable et appropriée (p. ex. une abstinence de consommation, une défense de possession d'arme, un couvrefeu) (Code criminel, art. 515[4]). Il faut mentionner ici que le non-respect de telles conditions est une infraction en soi, passible d'une peine allant jusqu'à deux ans d'emprisonnement (Code criminel, art.145), indépendamment du paiement de la caution éventuelle et de la révocation de la mise en liberté provisoire qu'il peut entraîner; et/ou 
- d'une libération appuyée par une personne garante qui s'engage à veiller au respect des conditions imposées au prévenu, sous peine de payer elle-même un montant d'argent que le juge établit.

Seuls trois motifs autorisent le juge à ordonner une détention provisoire (Code criminel, art. 515[10]) :

a) pour assurer la présence de l'accusé devant le tribunal. Selon la jurisprudence, la future présence du prévenu sera évaluée sur la base de son comportement préalable de conformité, de ses liens sociaux, de la nature de l'infraction, de la force de la preuve et de la peine potentielle encourue (Trotter, 1999);

b) pour assurer la protection ou la sécurité du public en général et des victimes en particulier, et protéger l'administration de la justice. Les critères jurisprudentiels pour évaluer la dangerosité du justiciable réferent à la nature et au contexte de l'infraction alléguée et ses suites, au profil social et mental de l'accusé, aux probabilités de sa condamnation et aux risques associés à sa libération (R.v. Rondeau, 1996);

c) pour ne pas miner la confiance du public envers l'administration de la justice, compte tenu notamment du caractère apparemment fondé de l'accusation, de sa gravité, des circonstances de la perpétration et de la longueur de la peine encourue.

Mais comme nous l'avons évoqué,il ne suffit pas de lire le Code criminel pour comprendre les pratiques des agents pénaux; sa mise en œuvre passe par leur activité (Noreau, 2000), sous-tendue par leur travail interprétatif. Nous examinons maintenant différentes dimensions de ces raisonnements.

\section{Le contexte sociopolitique : vers un principe de précaution généralisé?}

Les entretiens partagent le point de vue selon lequel, parmi ces trois motifs légaux pour requérir ou imposer une détention provisoire, c'est le second, celui de la protection et de la sécurité du public, qui est le plus invoqué. Le premier motif, qui vise 
à s'assurer de la comparution de l'accusé au procès, est certes également important mais, comme le commente ce magistrat :

"C'est rare que les gens soient prêts à [se soustraire à] la juridiction afin de pouvoir éviter un procès ou une accusation. » (Juge Tremblay)

De ce fait, ce motif ne serait pas souvent invoqué seul. Quant au troisième motif, celui de ne pas miner la confiance du public envers l'administration de la justice, un certain consensus se dégage aussi à son sujet, appuyé par la référence à la jurisprudence $(R$. v. Hall, 2002) : il semble peu populaire en raison de son fondement, étranger à l'accusé et à son comportement infractionnel passé ou futur. Selon les entretiens, il ne serait finalement valorisable que pour des situations exceptionnelles qui impliquent une violence très grave.

"Pour les magistrats, le motif de protection et de sécurité du public se concrétise d'abord dans l'objectif de préserver la collectivité d'événements impliquant de la violence physique. »
Pour les magistrats, le motif de protection et de sécurité du public se concrétise d'abord dans l'objectif de préserver la collectivité d'événements impliquant de la violence physique. Le contenu de cette notion est toutefois sujet à évolution, tant sur le plan social que politique : plusieurs entretiens mentionnent, par exemple, l'importance récente qu'ont pris des comportements comme la maltraitance intrafamiliale, ou encore la conduite avec facultés affaiblies, qui est porteuse de conséquences physiques potentielles. Au-delà de cette préoccupation pour la violence physique, il s'agit cependant aussi de prémunir le public contre des atteintes aux biens. Juges et procureurs de la poursuite le soulignent :

"Avoir quinze condamnations pour vol à l'étalage sur une période de quinze ans, puis là, il vient d'en faire trois. [...] Est-ce qu'on le remet en liberté parce que la sécurité du public n'est pas en danger? À mon point de vue, non.» (Juge Dubé)

"Est-ce que, parce que ce n'est pas un danger pour l'intégrité physique de la société, on ne devrait pas garder cette personne-là détenue? 
Je ne pense pas. Je pense que c'est mal en soi aussi pour la société. [...] Mon rôle, c'est que justice soit faite dans le système de justice. Et ma préoccupation primaire, c'est les victimes, c'est la société. [...] On est pour la paix sociale et la protection de la société. " (Maître Chartrand)

Le jugement moral contenu dans ce dernier extrait introduit en parallèle la mission que ce procureur attribue à la poursuite : celle de protéger la société. De façon intéressante, différents juges justifient une mise en détention provisoire par le même argument, en précisant peut-être plus fermement que sa mise en œuvre sera balisée par la jurisprudence et le droit :

«Je pense qu'évidemment la société doit se protéger contre certains individus, pas parce qu'on présume qu'ils vont être coupables, mais parce qu'il y a un potentiel de dangerosité... C'est pour ça que d'ailleurs, dans Rondeau, on encadre - en tout cas, on émet des critères qu'il faut regarder de façon attentive - pour s'assurer que si on le détient là, c'est vraiment parce qu'il n'y a pas d'autres solutions en attendant. " (Juge Roy)

"...il se dégage des entretiens comme de la littérature... une proximité entre les missions que les procureurs de la poursuite et les juges s'attribuent sur le thème de la protection de la société..."
De la sorte, et bien que les fonctions soient différenciées, il se dégage des entretiens comme de la littérature (Vanhamme, 2009) une proximité entre les missions que les procureurs de la poursuite et les juges s'attribuent sur le thème de la protection de la société, peut-être étoffée par le fait que la majorité des juges interviewés ont préalablement travaillé pour la Couronne. La fraternité entre cette mission et le second motif légal autorisant la détention provisoire est en outre textuellement évidente, ce qui contribue sans doute à la popularité de ce motif chez les magistrats. Ce qui semble aussi faciliter le recours à ce motif, c'est que l'argument de protection de la société, ou du public, semble pouvoir se décliner sous de nombreuses formes. En effet, la notion s'associe aussi très directement au problème du risque de récidive; le dernier extrait mentionné en témoigne. Les acteurs sont en ce sens appelés à 
jauger, aux fins de remise en liberté ou en détention provisoire, la probabilité de commission d'un nouvel acte infractionnel, et cette évaluation peut prendre racine dans les nombreux critères portant sur la dangerosité potentielle du prévenu ( $R$. v. Rondeau, 1996).

Ces critères concernent a priori une personne, en l'occurrence ses caractéristiques judiciaires, comportementales, psychiques ou sociales. Cependant, comme nous allons le voir, l'évaluation de la pertinence d'une remise en liberté n'est pas dénuée d'attaches contextuelles. Plusieurs juges mentionnent d'abord l'existence de directives hiérarchiques, de protocoles qui pousseraient les procureurs à la réserve face à une possibilité de requérir une remise en liberté. Ensuite et surtout, et probablement en lien avec ces consignes, la pression la plus communément soulignée est celle des médias. En effet, nous en rencontrons la mention dans les propos d'un grand nombre de personnes interviewées, qu'elles soient procureurs ou juges :

"Dès qu'il y a un drame qui se passe dans une région, tout le monde le sait. [...] On n'a pas peur, mais on est plus conscient de la réalité, peut-être $[\mathrm{du}]$ risque qu'on est prêt à prendre et peut-être à faire plus attention. " (Maître Gagnon)

«C'est aussi la surveillance sur tous les membres du processus maintenant, [...] la publicité autour du système judiciaire qui entre en jeu, la responsabilité qui est attachée à la personne qui prend la décision. Sauf pour le juge, parce que nous finalement, au niveau du juge, on est habitué de se faire critiquer sur nos décisions, ça fait partie de la fonction." (Juge Lavoie)

«Ça a amené les procureurs de la poursuite puis, dans une certaine mesure je pense, aussi les juges à être beaucoup plus prudents avant de remettre en liberté quelqu'un qui peut représenter un certain danger. » (Juge Roy) 
"...Webster, Doob

et Myers (2009)

ont constaté, dans

leur recherche, une

croissance significative

du nombre de cas

soumis aux tribunaux

ontariens en vue

de la détermination

d'une mise en liberté

ou d'une mise en

détention avant

jugement. »
Ces extraits laissent supposer qu'au moment de rendre une décision, certains juges, comme le premier, ne sont pas influencés par la pression médiatique, alors que d'autres, comme le second, lui accordent un certain effet de prudence. Quoi qu'il en soit, ces propos veulent souligner que les critiques des médias fuseraient, si une remise en liberté était suivie d'un dérapage. Un principe général de précaution s'en dégage alors, certainement plus exacerbé du côté de la poursuite :éviter d'avoir à se justifier d'une mise en liberté suivie de la commission d'un crime, alors qu'une mise en détention l'aurait empêchée. Cette préoccupation se situe certes aux niveaux individuel et professionnel du magistrat, celui de sa carrière, mais elle concerne aussi le niveau institutionnel de la justice, l'image de la justice dont se soucient autant les procureurs que les juges interviewés dans nos entretiens.

Dans ce schéma, le processus évaluatif du risque de récidive, qui porte a priori sur la personne de l'accusé, se révèle donc effectivement marqué par l'influence du contexte. En l'occurrence, il se colore des répercussions professionnelles et institutionnelles probables en cas d'une prédiction que des événements ultérieurs permettraient de qualifier d'infondée. Du côté des juges cependant, cette logique de prudence crée une tension avec les principes de droit qu'ils ont la charge de contrôler, dont le droit fondamental à la liberté, sur lequel nous reviendrons :

«C'est dans la Charte ça, le droit d'être remis en liberté.Alors, même si on n'avait pas l'article 510, 515(10) et puis qu'on voulait détenir le monde, il faudrait le justifier. C'est un aparté pour te montrer que très souvent, en matière de droit criminel, c'est une question de pondération, de balancement de différentes valeurs." (Juge Dubé)

Puisque ce principe de précaution s'infiltre dans les raisonnements, il faut aborder maintenant la question de ses effets. Dans le sens des propos exprimés ci-après par les personnes interviewées, Webster, Doob et Myers (2009) ont constaté, dans leur recherche, une croissance significative du nombre de cas soumis aux tribunaux ontariens en vue de la détermination d'une mise en 
liberté ou d'une mise en détention avant jugement. Or, dans l'ensemble des décisions répertoriées par ces auteurs, la proportion des décisions comportant le maintien en détention provisoire reste stable; donc, expliquent-ils, il en résulte nécessairement une croissance numérique des mises en détention. De plus, Webster (2009) a observé une nette tendance à la remise d'audience durant la procédure judiciaire ontarienne, tendance également évoquée par les magistrats de notre enquête. Cela signifie un accroissement du nombre moyen de comparutions avant la décision finale, dont il résulte un allongement de la durée de détention avant jugement (Kong et Peters, 2008; Casavant etValiquet, 2009). De nombreux juges relient plus ou moins explicitement la hausse du nombre des cas soumis au tribunal, ainsi que celle des comparutions en cour avant la décision, au principe de précaution que nous avons mentionné. Il induirait en effet un report de responsabilité qui imprégnerait toute la chaine des décisions avant celle du tribunal, un sentiment d'ailleurs confirmé du côté de la poursuite :

"Je peux comprendre le policier qui dit:

"Écoute, je n'ai pas de boule de cristal, on va laisser ça aux experts". Je la comprends cette décision-là. Puis j'oserais dire que la proportion de gens qui sont arrêtés, qui sont maintenant traduits devant le tribunal [...] a augmenté. Je pense que c'est devenu plus un réflexe. " (Juge Nadeau)

"À l'époque où j'étais procureur de la Couronne, on les prenait les décisions, on la prenait la chance, le risque, on assumait nos décisions. Aujourd'hui, les gens [...] sont peut-être moins prêts à prendre ce risquelà et préfêrent se faire valider par l'autorité. " (Juge Roy)

«On va laisser le juge décider; lui, il est là pour cela. Nous, on ne prendra pas cette chancelà. " (Maître Allard) 
"Cette « culture

d'évitement du

risque " ...peut

influer de la sorte, de façon directe, sur

l'augmentation de la population en détention provisoire. »
Selon ces propos, les policiers tendraient à laisser la responsabilité d'une mise en liberté dans les mains de l'avocat de la poursuite. Celui-ci, à son tour, sera plutôt porté à s'y opposer, ouvrant la voie à la tenue d'une audience d'enquête sur la mise en liberté. La Couronne exigera alors de la défense de sérieuses garanties pour prendre en considération une remise en liberté, ce qui peut allonger le délai de trois jours avant l'audience, ou favoriser des reports d'audience. Cette «culture d'évitement du risque " (Webster, Doob et Myers, 2009) peut influer de la sorte, de façon directe, sur l'augmentation de la population en détention provisoire.

D'une manière plus sinueuse, elle peut aussi mener à chercher les raisons pour lesquelles un accusé pourrait être libéré, plutôt qu'à s'interroger sur celles pour lesquelles il devrait être détenu. Ici encore, le contexte politique tendrait à renforcer cette orientation. En effet, on constate une multiplication des infractions associées à une présomption de détention provisoire, dont l'effet cognitif est mentionné par ce juge :

"Ça change la perception non seulement de la Cour, ça change la perception peut-être des avocats de la défense. C'est qu'on a besoin de démontrer à la Cour pour que vous soyez libéré... La loi a changé la façon dont c'est vu. » (Juge Ouellet)

Autrement dit, le principe de précaution qui sous-tend cette orientation législative peut favoriser une déviation au principe du droit fondamental à la liberté. Dans ce contexte, comment encore remettre en liberté?

\section{Le contexte organisationnel : les conditions d'une libération}

Lorsque les parties ne sont pas parvenues à un accord et se présentent à l'enquête sur la mise en liberté, la poursuite montre le cas échéant pourquoi, selon elle, un accusé ne serait a priori «pas 
" " La dangerosité, c'est difficile à évaluer, c'est la personne qui le sait de son intérieur s'il est dangereux ou pas. » (Juge Morin) 》 sortable " (Maître Bélanger). Sa plaidoirie fondée sur les motifs légaux de détention - dont principalement le second (énoncé plus haut) - portera par exemple sur le fait que le prévenu a un casier judiciaire lourd, qu'il est en rupture d'engagement, que le crime est grave, ces éléments pouvant se cumuler. La défense lutte alors contre cette vision et présente ses raisons et moyens pour soutenir une libération. Le juge à son tour examine les arguments présentés et, quel que soit le motif légal invoqué, il évalue la dangerosité potentielle de l'accusé (décrite plus haut). Pour ce faire, tout en se fondant sur les critères indiqués par la jurisprudence (cf.l'arrêt Rondeau), il mobilise toutes les ressources disponibles à l'audience. Celles-ci peuvent aller des plus objectives aux plus subjectives (Landreville et Laberge, 1992; Vanhamme, 2009), car la potentialité d'une dangerosité future reste difficile à cerner (Debuyst, 1984). L'évaluation qu'il en retire restera souvent voilée par un certain degré d'incertitude :

«La dangerosité, c'est difficile à évaluer, c'est la personne qui le sait de son intérieur s'il est dangereux ou pas." (Juge Morin)

"Puis des fois aussi, ça dépend du goût que tu as de prendre des risques pour des gens, ça peut être aussi bête que ça. Moi ça m'est arrivé fréquemment là, sur dossier, de voir qu'une personne devrait être détenue : tu appliques tes critères de l'arrêt Rondeau, ça milite tout en faveur d'une détention préventive. Mais pour une raison qui est difficilement articulable, là, tu penses que tu peux quand même te fier à la personne, tu as le goût de prendre un risque avec cette personne. » (Juge Gagnon)

Un juge peut ainsi se trouver face à un double dilemme au moment de décider du maintien ou non en détention : une relative incertitude sur le risque de récidive, d'une part, et la tension évoquée précédemment entre le principe de précaution et le droit à la liberté, d'autre part. Comme en réponse, tous les juges interviewés partagent une position de principe : ils 
"Une première exigence pouvant accompagner la libération est la caution en argent. » se disent tous favorables, dans la mesure du possible, à une libération accompagnée de conditions susceptibles d'en sécuriser le déroulement :

«S'assurer qu'on y va avec le grand principe que la remise en liberté, c'est le principe. L'exception, c'est la détention. [...] J'ai une preuve de continuité dans l'agir, puis il n'y a pas grandchose qui va changer dans son comportement. Ça fait que c'est en général la détention dans ce genre de dossier là... Encore là,je le répète, sauf dans des cas où il va y avoir un encadrement très solide. " (Juge Dubé)

Une première exigence pouvant accompagner la libération est la caution en argent. Destinée en principe à garantir la comparution aux audiences futures en référence au premier motif de détention, la caution prendrait souvent la forme d'un engagement. En concordance avec la jurisprudence (R. v. Garrington, 1973), le montant veut s'adapter à la situation pécuniaire du prévenu :

«"Combien êtes-vous en mesure d'offrir?". Des fois, les gens vont me dire :"Eh bien, je suis en mesure d'offrir 50 \$". Je vois si la personne me dit $[\ldots]$ : “Je suis fonctionnaire fédéral et je gagne 100000 \$ par année ”. Son 50 \$, je ne le prendrai pas au sérieux. Mais si c'est quelqu'un qui vit de prestations d'aide sociale, puis qu'il me dit : "C'est tout ce que j'ai à mettre sur la table", moi, je vais m'en satisfaire. Ce n'est pas tellement le montant qui est important pour moi : à mes yeux, c'est la façon dont la personne se positionne par rapport à cette hypothèse-là. " (Juge Tremblay)

Selon cet extrait, le processus d'établissement du montant de la caution sera aussi l'occasion de jauger la déférence ou la franchise de l'accusé, apportant de ce fait une indication globale sur la potentielle fiabilité de son comportement en cas de remise 
en liberté. La pratique étendrait donc l'application de la caution au motiflégal de prévention de la récidive. De nombreuses personnes interviewées mentionnent en outre qu'elle est très régulièrement imposée; ce fait n'est sans doute pas dénué de lien avec sa capacité d'être mobilisée pour évaluer ce risque.

En ce qui concerne les conditions qui accompagnent la libération, elles visent plus spécifiquement à définir un encadrement propice pour éviter ce risque. Certaines d'entre elles, dites négatives, imposent des restrictions et des interdictions (énoncées plus haut) et d'autres, dites positives, prescrivent un accomplissement dès lors que la défense y a consenti. Le sens qui est accordé à ces dernières conditions a souvent une visée de réhabilitation. Dans cette optique, le crime est considéré comme le résultat d'une inadaptation de l'individu, issue principalement de sa condition sociale. La condition est alors individualisée, vise la resocialisation, et sa nature est sociale ou psychologique. Une perspective réhabilitative peut en outre intégrer la question du risque que représente le justiciable, la resocialisation servant alors à prévenir ce risque (Gros, 2001). Dans nos entretiens, c'est du côté de la poursuite que cette dernière lecture ainsi que ses enjeux de précaution semblent plus fréquemment valorisés :

«On a tellement d'expérience à voir la difficulté des accusés et les embûches dans leur vie. [...] On essaie de trouver d'autres méthodes afin de pouvoir essayer de voir si on peut réussir à créer un changement. » (Juge Lavoie)

" Il $y$ a des gens peut-être qui, avec de l'aide, ne seraient pas un risque et qui peuvent être libérés." (Maître Bélanger) "

"Il y a des gens peut-être qui, avec de l'aide, ne seraient pas un risque et qui peuvent être libérés. " (Maître Bélanger)

" Ça enlèverait le fardeau aux avocats, aux procureurs de la Couronne de devoir assumer ce risque-là. » (Maître Gagnon)

Certaines de ces conditions positives d'encadrement peuvent, par exemple, porter sur le choix d'une résidence, la recherche d'un emploi, la reprise d'une formation. Dans les entretiens, c'est plutôt 
"...une majorité de magistrats regrettent que les services d'accompagnement social, en particulier ceux qui sont spécialisés dans ces problématiques, soient en sous-nombre." la question des conditions de suivi thérapeutique et rééducatif qui est développée, ce qui indique la présence de justiciables touchés par des problèmes de santé et de décrochage social. Parmi ceux-ci, les problèmes de dépendance et de déséquilibre mental semblent préoccuper particulièrement, et ce, en raison de la probabilité de récidive qu'ils entraîneraient :

"Même les procureurs de la Couronne sont prêts à accepter que, pour quelqu'un qui souffre de toxicomanie, si on se débarrasse du problème, on se débarrasse sans doute des infractions criminelles perpétrées par cette personne-là. » (Juge Lavoie)

" Il y a des cas où même si la personne n'a pas d'antécédents judiciaires, la Cour va quand même détenir à cause de la force de la preuve, de la gravité de l'infraction et du caractère bizarroïde de l'infraction commise. Là, on tombe dans la maladie mentale. Quand tu n'as rien qui te permet d'avoir un encadrement très, très, très clair, c'est tellement explosif comme situation et avec des conséquences tellement graves compte tenu de la nature du crime impliqué, que là, on va [le détenir].»(Juge Dubé)

À cet égard, une majorité de magistrats regrettent que les services d'accompagnement social, en particulier ceux qui sont spécialisés dans ces problématiques, soient en sous-nombre. Tous partagent l'idée qu'une offre plus large de ressources communautaires permettrait un meilleur encadrement de ces personnes et, de ce fait, éviterait certaines mises en détention, en permettant de prévenir certains dérapages grâce à un suivi spécialisé.

Un dernier type de condition est l'encadrement par une personne garante. Parce que la personne garante va être «les yeux et les oreilles de la Cour » (Juge Gagnon), elle doit présenter suffisamment d'indices de fiabilité. Son rôle est en effet de 
surveiller le prévenu, de contrôler s'il respecte adéquatement les conditions imposées, de le contraindre si possible à ce respect et de le dénoncer dans le cas contraire. Dans cette optique, pour tous les magistrats, l'idéal serait qu'elle soit un proche:

" Les meilleurs garants que tu peux avoir, c'est ton père ou ta mère. Mais si tu n'en as pas ou que tu as abusé de ces gens-là de ton propre milieu dans le passé, puis tu ne les as plus, tu es rendu avec des garants à distance. Le garant vient de [quartier éloigné] disons... Eh bien, c'est difficile pour la personne [depuis ce quartier éloigné] de superviser, hein? " (Juge Tremblay)

Les propos de certains juges évoquent cependant une certaine gêne envers la mobilisation de cette ressource, car elle pourrait placer la personne garante dans une situation de double contrainte. Dans le cas où la personne libérée ne se conformerait pas aux exigences du tribunal, son parent, qui s'est engagé comme garant par affection, risque en effet de se trouver face à une alternative où, quelle que soit la voie prise, son choix serait perdant : soit trahir son enfant, soit trahir son engagement envers la Cour. Le recours à une personne garante n'en reste pas moins prisé. Selon les entretiens, il deviendrait même la norme. La personne garante représente dès lors un instrument de renforcement des conditions qui densifie la mise en œuvre du principe de précaution.

Les conditions qui sont imposées sont estimées appropriées. Avocats de la poursuite et juges assurent non seulement que les conditions demandées ou données sont, par leur nature, adaptées spécifiquement à la situation du prévenu, mais aussi qu'elles sont toutes nécessaires au vu de celle-ci. Le fait qu'elles puissent finalement toutes être comprises en termes d'évitement du risque de récidive, met à nouveau en exergue l'importance de la logique de précaution dans les aspects concrets de la pratique cette fois. De tels raisonnements induisent alors qu'un prévenu ne se voit que rarement imposer une seule condition. Comme pour leur nature, leur nombre est estimé justifié en raison de l'encadrement nécessaire à la remise en liberté. Plusieurs juges ont mentionné à ce 
"...la multiplication

des conditions de libération aurait un effet implacable, celui d'une croissance des bris de conditions. " sujet que, pour établir les exigences requises pour une libération, ils utilisent un formulaire qui présente une liste de conditions à cocher. Bien que cette liste soit conçue pour faciliter le travail du tribunal, il ne nous semble pas impossible que le large éventail des choix qu'elle propose puisse, dans le même temps, inviter à l'octroi de plus d'une condition. En outre, les entretiens se rejoignent sur un emballement actuel du nombre de ces conditions :

"On a plus de conditions en raison des différentes politiques ministérielles qui ont été adoptées au fil des ans pour protéger davantage certains types de victimes. » (Juge Roy)

"La Couronne va dire dans les discussions de cubicule avec la défense: “Ton gars n'est pas fiable, pas confiance en lui, puis la dernière fois qu'on l'a mis en liberté, il a brisé ses conditions trois semaines plus tard et il a un historique de bris ", etc. Et là, la défense, pour contrer l'argument de la Couronne, va dire: "On va t'offrir un garant ". » (Juge Gagnon)

Or, la multiplication des conditions de libération aurait un effet implacable, celui d'une croissance des bris de conditions. Plusieurs participants le mentionnent :

« Les bris de conditions se multiplient. [...] La Couronne va pouvoir démontrer que la personne n'est pas fiable et ne respectera pas les conditions, donc ne devrait plus être libérée. Ça, c'est un pattern qu'on voit." (Juge Lavoie)

Selon les trois derniers extraits rapportant des propos tenus par des juges, la Couronne, en cas de non-respect de conditions, pourrait donc revendiquer avec une force accrue une mise en détention. En ce sens, si la multiplication des conditions de mise en liberté provisoire répondait au principe de précaution mentionné, elle créerait aussi les conditions justifiant son invocation, dans un mouvement centripète dont le centre serait la prison. 


\section{Le statut social face à la détention provisoire}

Ces trois extraits suggèrent en outre que la multiplication des conditions de libération découlerait directement de l'activité et de la responsabilité de la poursuite. Autrement dit, la décision du tribunal serait prise dans une configuration interactionnelle au sein de laquelle les arguments de la Couronne tendraient à jouir d'une prépondérance. Quelques procureurs ont d'ailleurs évoqué ce rôle influent :

«Au niveau de l'enquête de remise en liberté, évidemment, le juge a le pouvoir de prendre la décision, mais je réalise que c'est probablement l'étape dans le processus judiciaire où le procureur de la poursuite a le plus d'impact vraiment. Parce qu'à ce moment-là, nous, on a la connaissance du dossier, et le juge en prend connaissance très, très sommairement. [...] Alors, on est en position, disons, de donner une suggestion au juge qui soit plus éclairée. Donc, les juges vont avoir tendance, je pense - je peux me tromper, mais je pense - vont avoir tendance à plus suivre la suggestion du procureur de la poursuite, qu'ils ne le feraient au niveau d'un procès. " (Maître Chartrand)

Dans ce schéma, les conditions d'une libération — favorisée du côté des juges - se trouveront fortement soumises aux nécessités discernées par les avocats de la poursuite. Il est de ce fait intéressant d'explorer davantage ce qu'est, selon eux, un prévenu "sortable " ou non. Dans différents propos, et conformément à la littérature mentionnée ci-dessus, il apparait que l'évaluation a priori peut varier sur la base d'éléments extérieurs aux considérations légales :

«Deux personnes accusées du même crime, avec le même historique criminel ou avec les mêmes antécédents, [...] quelqu'un qui est sur le bienêtre social et qui habite au [Gîte X] [...], c'est 
" «Deux personnes accusées du même crime, avec le même historique criminel ou avec les mêmes antécédents, [...] quelqu'un qui est sur le bien-être social et qui habite au [Gîte $X][\ldots]$, c'est sûr que c'est vrai que cette personne-là n'a pas les mêmes garanties ou des garanties pour sortir, mais, dans son cas à lui, c'est possible que l'avocat ne fera même pas l'enquête de caution pour tenter de le faire sortir, [alors] que quelqu'un qui paye son avocat personnellement et que ce n'est pas un mandat de l'aide juridique, [...] il aura plus de chances de sortir." (Maître Bélanger) » sûr que c'est vrai que cette personne-là n'a pas les mêmes garanties ou des garanties pour sortir, mais, dans son cas à lui, c'est possible que l'avocat ne fera même pas l'enquête de caution pour tenter de le faire sortir, [alors] que quelqu'un qui paye son avocat personnellement et que ce n'est pas un mandat de l'aide juridique, [...] il aura plus de chances de sortir.» (Maître Bélanger)

"[Certains prévenus] ont des ressources, parce qu'ils sont capables de déposer 10000 \$, $15000 \$, 20000 \$$, il y a des gens qui vont venir témoigner pour eux, ils vont se présenter en cour en veston-cravate : ces gens-là vont être remis en liberté. » (Maître Chartrand)

"[Ceux-là sont] détenus par défaut, parce que justement il y a un manque de ressources, ils n'ont pas de famille, ils n'ont pas d'endroit où aller habiter, ils n'ont pas d'argent comme caution à fournir. " (Maître Nadon)

"Si on n'a pas d'interlocuteur avec qui discuter, on va demeurer sur notre position parce qu'on n'a pas plus d'informations. Mais plus on a d'informations, plus on peut être ouvert. " (Maitre Gagnon)

Selon ces extraits, le statut d'un prévenu semble en effet capable, dans certains cas, d'inverser une première évaluation de "pas sortable " par la voie des ressources sociales et pécuniaires qui le sous-tendent. Inversement, une première évaluation de "sortable" pourrait basculer, en raison des faibles ressources de l'accusé, vers un maintien en détention. En effet, certains justiciables sont maintenus en détention, alors qu'ils ne devraient a priori pas l'être.

Les propos ci-dessus font état d'une série d'éléments susceptibles de rendre une libération acceptable : avoir un bon défenseur et donc pouvoir se le payer; jouir par conséquent de revenus et donc 
"...le profil social des personnes fondé sur ces éléments renvoie aux caractéristiques de la classe moyenne, soit le revenu, l'aisance et le réseau social, qui semblent des privilèges bien éloignés des conditions de vie des moins nantis. " d'un travail au minimum; pouvoir de ce fait indiquer un domicile et une résidence stable; démontrer l'existence d'un entourage familial confiant et une intégration dans un réseau de personnes socialement valorisables; et offrir grâce à eux des témoignages favorables et convaincants. On pourra constater que le profil social des personnes fondé sur ces éléments renvoie aux caractéristiques de la classe moyenne, soit le revenu, l'aisance et le réseau social, qui semblent des privilèges bien éloignés des conditions de vie des moins nantis. Le fait que ceux-ci aient un faible capital en ce qui concerne les attributs liés au statut social, limiterait donc leur capacité à répondre aux exigences requises pour être libérés provisoirement, puisque ces exigences se fondent justement sur un profil qui n'est pas le leur. De plus, cette configuration qui leur est défavorable pourrait s'emballer sous la pression des tendances actuelles que notre enquête a soulignées. Plus nombreuses seraient les exigences pour la libération d'une personne dépourvue de ressources pécuniaires et sociales, moins celle-ci aurait de possibilités d'y répondre, et donc de chances d'être libérée. Si elle était libérée, compte tenu des lacunes d'accompagnement social et thérapeutique mentionnées et de l'isolement lié souvent au décrochage social, le nombre élevé de conditions, conjugué à la faiblesse du soutien mobilisable, augmenterait les probabilités de non-respect des conditions. S'il s'agissait alors de chercher les raisons pour lesquelles elle pourrait être remise en liberté après un bris de condition, le peu de garanties qu'elle aurait encore à offrir minerait ce projet. Ces logiques, comme nous l'énoncions en introduction, peuvent interpeller le droit pour tous, hors juste cause, à une mise en liberté assortie d'une caution raisonnable.

\section{Conclusion}

C'est de longue date que la recherche a montré l'intervention de critères extra-légaux dans le processus décisionnel judiciaire (cf. Vanhamme et Beyens, 2007). La présente contribution montre que la détermination de la détention provisoire, comme celle de la peine, s'avère ainsi un processus humain (Hogarth, 1971) 
"...un traitement

différencié, dans

lequel les personnes

vulnérables risquent

davantage une mise en détention, prend quelques-unes de ses racines à l'intérieur du système pénal qui se révèle lui-même soumis aux conditions du contexte dans ses dimensions sociales et politiques. » et une pratique sociale (Hutton, 2006), dans lesquels le fait de ressortir des couches sociales fragiles peut nuire à l'accusé. En effet, dans un contexte sociopolitique marqué par l'inquiétude sécuritaire, l'évaluation de la dangerosité future apparait au cœur des raisonnements. En raison de l'incertitude que la notion soulève, celle-ci appelle nécessairement à la mobilisation de critères extralégaux (que l'arrêt Rondeau tente "d'objectiver»). Ensuite, dans un contexte organisationnel qui valoriserait les ressources sociales de la classe moyenne, l'accusé qui en est dénué serait identifié pour sa différence et aurait, de surcroît, moins à offrir pour appuyer sa libération. Enfin, dans un contexte institutionnel marqué par un principe de précaution, l'accusé risquerait davantage de rester en détention et, s'il était libéré, il risquerait réellement de ne pas respecter l'une des multiples conditions qui lui seraient imposées. C'est en ce sens que nous avons pu soutenir qu'un traitement différencié, dans lequel les personnes vulnérables risquent davantage une mise en détention, prend quelques-unes de ses racines à l'intérieur du système pénal qui se révèle luimême soumis aux conditions du contexte dans ses dimensions sociales et politiques.

Le principe de précaution qui traverse les discours recueillis concerne l'évitement du risque, à la fois de récidive, de mise en cause de la responsabilité du professionnel et de délégitimisation de l'institution de la justice. À la racine de ces préoccupations réside une urgence de préservation sociale - de son corps et de son ordre - , qui a été arguée et réitérée depuis le mouvement de défense sociale à l'aube du XX ${ }^{\mathrm{e}}$ siècle (Prins, 1910) jusqu'aux pratiques actuelles de la nouvelle pénélogie (Feeley et Simon, 1992, 1994; Simon et Feeley, 2003). De telles inquiétudes se fondent sur la peur qui émerge dans une société (ultra)libérale sous tension permanente, dans laquelle les valeurs dominantes se trouvent sans cesse mises au défi (Tulkens, 1986; Mary, 2001). Dans cette configuration de repli, les groupes sociaux les plus marginaux risquent forcément de se retrouver dans la mire du contrôle social et pénal (Simon, 1993; Simon et Feeley, 2003). 


\section{Notes}

1 En 1989-1990, on dénombrait en moyenne, par jour, 4702 prévenus et 12986 condamnés dans les prisons des provinces et territoires; les personnes en détention provisoire constituaient ainsi $27 \%$ du total de 17688 détenus. En 2013-2014, on y dénombrait 11493 prévenus et 9888 condamnés; les personnes en détention provisoire représentaient $54 \%$ du total de 21381 détenus (Statistique Canada, CANSIM, Services correctionnels pour adultes. Comptes moyens des contrevenants dans les programmes provinciaux et territoriaux). En 25 ans, le nombre des détentions avant jugement a donc crû de 6701 (+144\%), alors que celui des détentions après condamnation a décru de 3098 (24\%). La conjonction de ces deux mouvements inverses produit la part maintenant majoritaire de ces détentions avant jugement dans la population pénitentiaire. Bien sûr, toutes les provinces ne contribuent pas similairement à cette tendance, ni pour le nombre de personnes incarcérées, ni pour le pourcentage de prévenus dans cette population.

2 La détention avant jugement, pratiques et logiques pénales et sociales, subvention du Conseil de recherches en sciences humaines du Canada.

3 Ces provinces ne sont pas nommées afin de protéger au mieux l'anonymat des personnes interviewées qui évoluent dans une communauté professionnelle relativement restreinte, ainsi que la confidentialité des expériences, avis et analyses qu'elles nous ont livrés avec une grande confiance. C'est avec ce même souci que les extraits d'entretiens présentés dans la suite de l'article sont associés à des noms fictifs.

4 Pour plus de détails sur les dispositifs méthodologiques et les stratégies d'analyse de ces différents travaux, voyez : Labelle, 2012, p. 32-48; Labelle etVanhamme, 2015, p. 84-86; etVanhamme, 2015, p. 66-68.

\section{Bibliographie}

BEYENS, Kristel (2000). Straffen als sociale praktijk. Een penologisch onderzoek naar straftoemeting, Brussel, VUBPress.

CASAVANT, Lyne, et Dominique VALIQUET (2009). Projet de loi C-25 : Loi sur l'adéquation de la peine et du crime, Ottawa, Bibliothèque du Parlement du Canada, Service d'information et de recherche parlementaires.

CICOUREL, Aaron V. (1979). La sociologie cognitive, Paris, PUF.

CONSEIL NATIONAL DU BIEN-ÊTRE SOCIAL (2000). La justice et les pauvres, Ottawa, Travaux publics et Services gouvernementaux Canada.

COUSINEAU, Marie-Marthe (1995). «La détention provisoire au Québec :éléments de connaissance et propositions de réflexions ", Criminologie, Vol. 28, № 2, p. 5-26.

CROCKER, Anne G., et collab., (2007). «Rate and characteristics of men with an intellectual disability in pre-trial detention ", Journal of Intellectual \& Developmental Disability, Vol. 32, № 2, p. $143-152$.

DEBUYST, Christian (1984). "La notion de dangerosité, maladie infantile de la criminologie ", Criminologie, Vol. 17, № 2, p. 7-24. 
FEELEY, Malcolm M., et Jonathan SIMON (1992). «The New Penology: Notes on the Emerging Strategy of Corrections and Its Implications ", Criminology, Vol. 30, № 4, p. 449-474.

FEELEY, Malcolm M., et Jonathan SIMON (1994). «Actuarial Justice: the Emerging New Criminal Law ", dans David Nelken (dir.), The Futures of Criminology, London, Sage, p. 173-201.

FRIEDLAND, Martin L., (1965). Detention before Trial: A Study of Criminal Cases Tried in the Toronto Magistrate's Court, Toronto, University of Toronto Press.

GARCEAU, Marie-Luce (1990). «La détention provisoire au Québec : une pratique judiciaire courante », Criminologie, Vol. 23, № 1, p. 117-134.

GEERTZ, Clifford (1998). "La description dense.Vers une théorie interprétative de la culture ", Enquête. Archives de la revue Enquête, № 6, p. 73-105.

GROS, Frédéric (2001). "Les quatre foyers de sens de la peine », dans Antoine Garapon, Frédéric Gros et Thierry Pech, Et ce sera justice. Punir en démocratie, Paris, Odile Jacob, p. 11-138.

HOGARTH, John (1971). Sentencing as a Human Process, Toronto, University of Toronto Press.

HUTTON, Neil (2006). «Sentencing as a Social Practice », dans Sarah Armstrong et Lesley McAra (dirs.), Perspectives on Punishment. The Contours of Control, Oxford University Press, p. 155-174.

JOHN HOWARD SOCIETY OF ALBERTA (2009). An analysis of the issue of "delay" for sentencing purposes by accused held in remand, www.johnhoward.ab.ca/pub/timeserved/credit-analysis.pdf

KELLOUGH, Gail (1996). " 'Getting Bail': Ideology on Action », dans Thomas O’Reilly-Fleming (dir.), Post-Critical Criminology, Toronto, Prentice-Hall, p. 159-183.

KELLOUGH, Gail, et Scot WORTLEY (2002). «Remand for plea: The impact of race, pre-trial detention and over-charging on plea bargaining decisions ", British Journal of Criminology, Vol. 42, No 1, p. 186-210.

KONG, Rebecca, etValerie PETERS (2008). «Tendances de la détention provisoire dans les services correctionnels pour adultes et la détermination de la peine ", Juristat, Centre canadien de la statistique juridique, Statistique Canada, Vol. 28, № 9, 13 p.

KOZA, Pamela, et Anthony N. DOOB (1974). "Some Empirical Evidence on Judicial Interim Release Proceedings ", Criminal Law Quarterly, Vol. 17, p. 258-272.

LABELLE, Vicky (2012). Une peine sans coupable : la détention provisoire, Mémoire de maîtrise non publié, Département de criminologie, Faculté des sciences sociales, Université d'Ottawa.

LABELLE,Vicky, et Françoise VANHAMME (2015). "Les risques du métier de Procureur ", dans Marion Vacheret et Fernanda Prates (dirs.), La détention avant jugement au Canada : Une pratique controversée, Montréal, Presses de l'Université de Montréal, p. 65-82.

LANDREVILLE, Pierre, et Danielle LABERGE (1992). "Détention sous garde et dangerosité ", Les cahiers du GRAPPP, Nº12, Université de Montréal / Université du Québec à Montréal.

LESSARD-HÉBERT, Michelle, Gabriel GOYETTE et Gérald BOUTIN (1997). La recherche qualitative. Fondements et pratiques, Paris, Bruxelles, De Boeck Université, Méthodes en sciences humaines.

MCLEAN, Julia (1995). "Prévenues et détenues logées à la même enseigne, l'exemple des prisons de Burnaby et Tanguay ", Criminologie,Vol. 28, Nº 2, p. 43-60.

MARY, Philippe (2001). «Pénalité et gestion des risques : vers une justice "actuarielle" en Europe?", Déviance et société, Vol. 25, № 1, p. 33-51. 
NOREAU, Pierre (2000). "Judiciarisation et déjudiciarisation : la part de la poursuite et de la défense ", Criminologie,Vol. 33, № 2, p. 55-79.

PIRES,Alvaro (1997). «Échantillonnage et recherche qualitative : essai théorique et méthodologique ", dans Jean POUPART, Jean-Pierre DESLAURIERS, Lionel-Henri GROULX, Robert Mayer et Alvaro Pires, La recherche qualitative. Enjeux épistémologiques et méthodologiques, Montréal, Gaëtan Morin, p. 113-169.

PORTER, Lindsay, et Donna CALVERLEY (2011). "Tendances de l'utilisation de la détention provisoire au Canada ", Juristat, No 85-002-X, Statistique Canada.

PRINS, Adolphe (1910/1986). La défense sociale et les transformations du droit pénal, Genève, Médecine et Hygiène, coll. «Classiques Déviance et Société ».

SIMON, Jonathan (1993). Poor Discipline. Parole and the Social Control of the Underclass, 1890-1990, Chicago, London, University of Chicago Press.

SIMON, Jonathan, et Malcolm M. FEELEY (2003). "The Form and Limits of the New Penology ", dans Thomas Blomberg et Stanley Cohen (dirs.), Punishment and Social Control, New York, Aldine de Gruyter, p. 75-116.

STATISTIQUE CANADA. Services correctionnels pour adultes, comptes moyens des contrevenants dans les programmes provinciaux et territoriaux, Tableaux détaillés de CANSIM, http://www5.statcan.gc.ca/ subject-sujet/subtheme-soustheme.action?pid=2693\&id=2149\&lang=fra\&more $=0$

SUDNOW, David (1965). " Normal Crimes. Sociological Features of the Penal Code in a Public Defender's Office ", Social Problems, № 12, p. 255-276.

TROTTER, Gary (1999). The Law of Bail in Canada, Toronto, Thomson Canada.

TULKENS, Françoise (1986). «Un chapitre de l'histoire des réformateurs. Adolphe Prins et la défense sociale ", dans Adolphe Prins (1910/1986), Op. Cit., p. 17-46.

VANHAMME, Françoise (2009). La rationalité de la peine : enquête au tribunal correctionnel ", Bruxelles, Bruylant, Coll. de l'École des sciences criminologiques Léon Cornil.

VANHAMME, Françoise (2015). «Les conditions judiciaires du maintien en liberté », dans Marion Vacheret et Fernanda Prates (dirs.), La détention avant jugement au Canada : une pratique controversée, Montréal, Presses de l’Université de Montréal, p. 83-104.

VANHAMME, Françoise, et Kristel BEYENS (2007). "La recherche en sentencing : un survol contextualisé ", Déviance et société, Vol. 31, № 2, p. 199-228.

WEBSTER, Cheryl M. (2009). «Out of Sight, Out of Mind: A Case Study of Bail Efficiency in an Ontario Video Remand Court ", Current Issues in Criminal Justice, Vol. 21, No 1, p. 103-126.

WEBSTER, Cheryl M.,Anthony N. DOOB et Nicole MYERS (2009). «The Parable of Ms Baker:

Understanding Pre-Trial Detention in Canada ", Current Issues in Criminal Justice,Vol. 21, p. 79-102.

R. v. Garrington, 1973, 1 O.R. 370, (1972), 9 C.C.C. (2d) 472 (H.C.J.).

R. v. Rondeau, 1996, R.J.Q. 115,108 C.C.C. (3d) 474.

R. v. Hall, 2002, 3 S.C.R. 309, 2002 SCC 64 